\title{
Percepciones sobre la Gestión del Conocimiento de Directivos Universitarios de Cuatro Universidades Chilenas
}

\author{
Emilio R. Rodríguez-Ponce ${ }^{(1)}$ y Liliana M. Pedraja-Rejas ${ }^{(2)}$ \\ Universidad de Tarapacá, (1) Instituto de Alta Investigación; (2) Centro de Estudios CEUTA, 18 de \\ Septiembre 2222, Arica-Chile. (e-mail: emilior.rodriguez.ponce@gmail.com; Ipedraja@uta.cl)
}

Recibido Sep. 25, 2015; Aceptado Ene. 5, 2016; Versión final Feb. 24, 2016, Publicado Ago. 2016

\begin{abstract}
Resumen
Este estudio tiene como objetivo explorar las percepciones de directivos de cuatro universidades chilenas sobre el proceso de gestión del conocimiento. Para lograr dicho objetivo se aplica un cuestionario a 34 directivos de cuatro universidades chilenas. Así, se midió cada fase de la gestión del conocimiento mediante una escala de Likert de 1 a 7 . Los resultados de la muestra indican una correlación significativa entre las fases de la gestión del conocimiento (crear, compartir y aplicar), lo que sugiere que se trata de un proceso inclusivo, donde cada una de las fases no puede considerarse de forma aislada. Se descubre también que la creación de conocimiento (3.897) y el intercambio de conocimiento (4.194) tiene un promedio, en la muestra empleada, que no es significativamente diferente de la mediana (4.000) de la escala utilizada ( $p$ $<0.05)$. En la etapa de aplicar el conocimiento se encuentra un promedio $(3,524)$ significativamente menor que la mediana (4.000) de la escala utilizada ( $p>0.05)$. Por consiguiente, los resultados del estudio sugieren que la etapa más complicada de abordar en el proceso de gestión es aplicar dicho conocimiento para generar valor estratégico a la institución.
\end{abstract}

Palabras clave: gestión del conocimiento; universidades; educación superior.

\section{Perceptions about Knowledge Management from Top Managers of four Chilean Universities}

\begin{abstract}
This study aims to explore the perceptions of managers from four Chilean universities about the process of knowledge management. To achieve this goal a questionnaire was applied to 34 managers from four Chilean universities. Thus, each phase of knowledge management was measured using a Likert scale from 1 to 7 . Results indicate that there is a significant correlation between the phases of knowledge management (creating, sharing and applying), suggesting that this is an inclusive process where each phase cannot be isolated considered. Moreover, it was found that creating knowledge (3.897) and sharing knowledge (4.194) has an average, in the sample employed, that is not significantly different from the median (4.000) of the scale used $(p<0.05)$. In the step of applying knowledge it was found an average (3.524) that is significantly lower than the median (4.000) of the scale used ( $p>0.05$ ). Consequently, this study suggests that the most complicate step in the phase of applying knowledge is to generate strategic value from knowledge management.
\end{abstract}

Keywords: knowledge management; universities; higher education. 


\section{INTRODUCCIÓN}

En Chile, existe una brecha entre los roles que la sociedad del conocimiento demanda de las universidades y la calidad con la cual estas organizaciones responden a dichas demandas. Lo anterior implica reconocer que los niveles de calidad de las instituciones chilenas son muy diversos y sólo una cantidad menor de instituciones cumple a cabalidad con todas las tareas requeridas para las entidades universitarias en la denominada nueva economía (Rodríguez-Ponce 2009). Por lo tanto, es relevante identificar qué factores o aspectos son los que permiten que unas universidades se diferencien radicalmente de otras en sus niveles de desempeño y calidad.

Desde esta perspectiva, siguiendo la lógica de la teoría de recursos y capacidades (por ejemplo; Wernerfelt, 1984; Prahalad y Hamel, 1990; Amit y Shoemaker, 1993), se puede postular que existe un vínculo ineludible entre los recursos institucionales y los niveles de calidad brindados por las universidades (Araneda et al., 2013). Pero la evidencia también muestra que instituciones de tamaño similar, con recursos equivalentes, siguen derroteros estratégicos distintos y, consecuentemente, alcanzan diferentes niveles de calidad, en el desarrollo de su quehacer institucional.

Ciertamente, las capacidades tienen impacto en el desempeño organizativo y las capacidades no sólo se asocian a recursos sino que también a procesos de dirección y gestión. El punto a plantear es que los equipos de dirección adoptan decisiones y son las decisiones las que definen el camino a seguir y determinan el nivel de éxito organizacional. En efecto, en una sociedad como la actual, caracterizada por altos niveles de: competencia, dinamismo, y complejidad, donde existen grandes stocks y flujos de información y conocimiento; el papel de la gestión del conocimiento al interior de los equipos directivos, no sólo puede ser un determinante estructural de las decisiones, sino que estas decisiones pueden incidir significativamente en el nivel de calidad que alcancen las universidades (Rodríguez, 2012). En dichos procesos decisionales, los equipos gestionan conocimiento. Esto es crean, comparten y aplican conocimiento para adoptar e implementar las mejores decisiones. Por consiguiente, el proceso de toma de decisiones es una instancia de creación de valor organizacional.

Ahora bien, las instituciones universitarias que tienen como tarea la creación de conocimiento para el desarrollo sustentable deberían ser un ejemplo de creación de valor a su interior (López et al., 2015). Dada esta situación parece relevante explorar la percepción acerca del proceso de gestión del conocimiento, al interior de los equipos de dirección de las unidades académicas, ya que dicho proceso puede incidir sobre la calidad del quehacer institucional, en las respectivas unidades académicas de las universidades. En consecuencia, en la presente investigación, se tratará de explorar las percepciones de los directivos respecto de los actos de crear, compartir y aplicar conocimiento organizacional. Se trata de dar un primer paso, de carácter exploratorio, para validar empíricamente las variables empleadas y para dar luz acerca de una materia poco estudiada en el campo de las universidades en países emergentes como Chile.

\section{MARCO TEÓRICO}

El estudio de la gestión del conocimiento en las organizaciones se puede abordar bajo diferentes perspectivas tales como la que proporciona la sociología de las organizaciones o la psicología organizacional. Sin embargo, en esta investigación se considera el enfoque de la economía de las organizaciones y se asume que las universidades son un tipo de organización, en las que como lo indica Coase (1937) existen vínculos permanente más allá de las relaciones de mercado entre los agentes que la conforman; agentes económicos que por cierto poseen una racionalidad limitada (Simon, 1979) y cuyas decisiones, además, pueden ser oportunistas sin los debidos resguardos estructurales y legales (Williamson, 2002).

Por consiguiente, bajo la existencia de relaciones permanentes entre los agentes económicos de una universidad, más allá de las posibles transacciones de mercado, en presencia de determinadas estructuras organizativas, se debe reconocer como lo sostienen Hambrick y Mason (1984) que en un contexto de racionalidad limitada la base cognitiva y los valores de la alta dirección limitan el campo de la visión, influyendo sobre la percepción selectiva, la interpretación y, consecuentemente, en la selección de la estrategia. Es decir, las decisiones adoptadas pueden ser diferentes dependiendo de las características particulares del equipo de dirección. Por lo mismo, resulta fundamental conocer cómo se gestiona el conocimiento en los equipos directivos y cómo los procesos de crear, compartir y aplicar conocimiento pueden impactar en la toma de decisiones y en los resultados de las entidades educativas.

En consecuencia, en este estudio exploratorio se trata de indagar las percepciones que sobre el proceso de gestión del conocimiento tienen los directivos de 4 universidades. Ciertamente, se trata de un estudio exploratorio que no establece relaciones causales ni permite realizar inferencia estadística, pero que sí 
permite inducir a partir de los hallazgos (Snow y Thomas, 1994) una perspectiva global del proceso de gestión del conocimiento.

En virtud de lo anterior, esta discusión bibliográfica se sustenta en los avances teóricos y prácticos que se han desarrollado respecto de la gestión del conocimiento en el campo de la economía de las organizaciones. En este sentido, se sigue la lógica de Dalkir (2013), considerando como elementos centrales de la discusión lo que es el conocimiento y el proceso de gestión del conocimiento asociado a: crear, compartir y aplicar conocimiento.

El conocimiento es la comprensión, conciencia y familiaridad acerca de un tema, tópico o aspecto, adquirida a través del estudio, investigación, observación o experiencia en el curso del tiempo (Smith y Rupp, 2002). Para una organización, el conocimiento se puede definir como lo que la gente sabe acerca de los productos, clientes, procesos, errores y éxitos. Esto implica que el conocimiento consiste en la habilidad para sustentar el despliegue coordinado de los recursos y las capacidades para contribuir al logro de los propósitos y metas institucionales (Patel y Gorvadiya, 2014). En la literatura se reconoce que el conocimiento se expresa de 3 formas de capital intelectual (Wang et al., 2014): el capital humano, el capital estructural y el capital de relaciones comerciales.

El Capital Humano se refiere al conocimiento, habilidades y talentos disponibles por parte de los miembros de una organización. El Capital Estructural se refiere al sistema organizativo establecido para codificar, transmitir y aplicar conocimiento. El Capital de las Relaciones Comerciales surge de los contactos y conexiones creadas con los clientes, consumidores, vendedores y proveedores. Por su parte, existen 2 tipos diferentes de conocimiento (Grant, 1996): el conocimiento explícito y el conocimiento tácito.

El Conocimiento Explícito, es aquel que está disponible para todos los miembros de la organización. Se trata de un conocimiento claramente formulado y fácilmente expresable, sin ambigüedad. Se dispone en las bases de datos y los sistemas organizacionales y en tal sentido, puede ser compartido y comunicado fácilmente entre los miembros de la organización. Dicho conocimiento se puede dividir en 3 fuentes (Patel y Gorvadiya, 2014); Conocimiento cognitivo, referido al "Know what", que considera la maestría básica en una disciplina obtenida a través del entrenamiento y la experiencia; Habilidades avanzadas, referido al "Know how", la habilidad para aplicar reglas y/o aprender a solucionar problemas del mundo real, relacionada a la capacidad para aplicar conocimientos en la solución de problemas de alta complejidad; Sistemas de entendimiento o expertos, referido al "Know why", relacionado con la comprensión profunda de las relaciones, causas y efectos de una situación o disciplina en particular. El Conocimiento Tácito, es el conocimiento no articulado que está en la mente de las personas. Está en los individuos y no es fácil de expresar en palabras. Se asocia a las lecciones aprendidas, la experiencia ganada, la experiencia recibida.

Ahora bien, en la nueva economía, el conocimiento constituye la fuente esencial de la ventaja competitiva de las organizaciones, ya que el capital intelectual individual y colectivo es el recurso principal del que disponen las instituciones para llevar a cabo su cometido. En tal contexto, las organizaciones buscan las estrategias que permitan a sus empleados compartir experiencias, impresiones y conocimientos, bajo la premisa que en dicho proceso se construye el valor estratégico (Rodríguez-Ponce et al., 2014). En efecto, la gestión del conocimiento es un proceso insoslayable y un determinante del éxito tanto en firmas de alta tecnología (Martín de Castro, 2015), como en organizaciones de servicios (Kumar, 2010) e incluso en instituciones sin fines de lucro (Hume et al., 2012).

En la sociedad del conocimiento el acto de crear y compartir conocimiento es la base de la ventaja competitiva (Lee y Chen, 2012). De hecho, se ha descubierto que la gestión del conocimiento genera innovaciones que llevan a mejores resultados económicos para las firmas (Alegre et al., 2013); pero no sólo las innovaciones son la resultante de la gestión del conocimiento, sino que también lo es el diseño de la estrategia. Es decir, la gestión del conocimiento permite diseñar mejores procesos estratégicos impactando en el desempeño de las organizaciones por esta vía (Martins y Miranda, 2011). Con todo, la gestión del conocimiento no se genera de manera espontánea, sino que responde al grado de colaboración que puede lograrse al interior de los equipos de trabajo. El grado de colaboración, a su vez, se asocia de manera indisoluble con la cultura organizativa, ya que existirán ambientes más o menos propicios para la colaboración al interior de los equipos de dirección (Rodríguez-Gómez y Gairín, 2015).

En consecuencia, el trabajo colaborativo se vincula a la sinergia que se establece entre individuos o grupos de individuos mediante una dinámica de trabajo adecuada. La cuestión es que los individuos juntos pueden alcanzar mayores o mejores logros de los objetivos predefinidos, que de forma no colaborativa (Gairín, 2015). Por ende, es posible esperar que la gestión del conocimiento sea diferente en distintas organizaciones y sus impactos sobre la calidad institucional y el desempeño sean de distinta magnitud y 
sentido. Desde esta perspectiva, Rodríguez-Gómez y Gairín (2015) sugieren que el acto de promover e impulsar el trabajo colaborativo permite que las organizaciones se constituyan en construcciones sociales al servicio de las personas y del entorno en el que interactúan.

La gestión del conocimiento es un proceso que permite la creación de conocimiento, para luego compartir ese conocimiento entre los miembros de una organización, y aplicarlo para generar innovaciones o mejoras en los productos o servicios, pero también en la adopción de decisiones e ideas que buscan el mejoramiento de las instituciones. La gestión del conocimiento transforma a éste en un activo estratégico, que genera ventaja competitiva y aprendizaje en las organizaciones (Zhao et al., 2012). En esta centuria de creciente competencia y exigencias de innovación de productos, la creatividad y las ideas son fundamentales para que las organizaciones respondan a los requerimientos. Por consiguiente, los recursos organizativos más valiosos son las habilidades, la experticia y las relaciones del personal, focalizados en la perspectiva de gestionar el conocimiento para crear valor a la organización (Geisler y Wickramasinghe, 2015). Así, el proceso del conocimiento consiste en la búsqueda y la combinación de sinergias, a partir de la información y la capacidad de procesamiento, creatividad e innovación de quienes gestionan la información, la comparten y la aplican creando conocimiento. Desde esta perspectiva, el proceso de gestión del conocimiento comprende 3 etapas (Pedraja-Rejas et al., 2006; Nonaka y Takeuchi, 1995):

\section{Crear conocimiento}

La creación de conocimiento es un desafío que conlleva a que las organizaciones creen nuevas ideas desde las personas que toman las decisiones (Stone, 2010). Este es un proceso esencialmente cognitivo que implica la exploración, combinación y el descubrimiento de conocimiento (Goldman y Scardamalia, 2013). Ciertamente, los individuos que conforman una organización crean conocimientos nuevos a través de conexiones intuitivas entre las ideas existentes, y/o a través de la interacción con otros individuos de la organización en procesos basados en la explicación del conocimiento y diálogo (Chaudhri et al., 2013).

\section{Compartir conocimiento}

El proceso de compartir conocimiento, se genera cuando unos individuos transfieren conocimientos a otros. Así, el compartir conocimiento es posible y necesario para lograr intercambios que generen un proceso de creación de valor en la gestión del conocimiento (Ritala et al., 2015). El conocimiento generado es poder sólo en la medida que se comparta al interior de las organizaciones (Chong y Besharati, 2014). Por lo tanto, el desafío en los equipos directivos consiste, entonces, en compartir información y conocimiento de forma tal que los miembros del equipo puedan integrar ese conocimiento a favor de la institución (Osmani et al., 2014).

\section{Aplicar conocimiento}

El proceso final en la gestión del conocimiento, es aplicarlo, es decir, convertir el conocimiento en productos valiosos para la organización (Osei-Bryson et al., 2014). La aplicación del conocimiento se facilita, a través de sistemas dinámicos y flexibles que permiten compartir ideas y convertirlas en productos o servicios (Patel y Gorvadiya, 2014). Dicha aplicación de conocimiento no sólo produce bienes reales sino que también activos intangibles cuyo verdadero impacto es muy difícil de valorar (Hsieh et al., 2014). Naturalmente, la gestión del conocimiento debe entenderse como un proceso integrador, más que como una sumatoria de fases aisladas. De hecho la evidencia en Chile muestra que en las pequeñas y mediana empresas (PedrajaRejas y Rodríguez-Ponce, 2008) y en organizaciones públicas (Rodríguez-Ponce, 2007), existen altos grados de correlación entre las fases de crear, compartir y aplicar conocimiento.

Se ha probado empíricamente que la aplicación del conocimiento es una fase determinante del éxito en la gestión del conocimiento (Sarin y McDermott, 2003), y su culminación es difícil ya que un proceso previo de crear y compartir conocimiento es condición necesaria pero no suficiente para aplicar conocimiento con éxito (Choi et al., 2010). Lo anterior, no debe hacer perder de vista que sin las fases de crear y compartir conocimiento, es imposible aplicarlo en un modo que impacte positivamente sobre la eficacia o eficiencia organizativa (Lee et al., 2005).

\section{METODOLOGÍA}

\section{Participantes y procedimiento de recolección}

Un total de 34 directivos de 4 distintas universidades chilenas participaron en el estudio. Todos los participantes cumplieron con los siguientes criterios: (1) Ser directivo superior o directivo académico de una universidad acreditada; (2) Trabajar en la modalidad de jornada completa en dicha universidad; (3) Tener labores de dirección en una unidad académica que adopta decisiones relevantes en forma autónoma; (4) 
Disponibilidad para participar voluntariamente del estudio. Para el procedimiento de recolección se utilizó un cuestionario adaptado de Rodríguez-Ponce (2007), diseñado específicamente para este estudio exploratorio, el cual contiene 14 preguntas y se puede observar en la Tabla 1.

Tabla 1: Cuestionario diseñado para este estudio exploratorio

\begin{tabular}{|l|l|}
\hline \multicolumn{2}{|l|}{ Identificación de la institución } \\
\hline Nombre & \multicolumn{2}{|l|}{} \\
\hline Dirección & \\
\hline Datos del encuestado \\
\hline Persona que responde \\
\hline Cargo \\
\hline Instrucciones para responder \\
\hline $\begin{array}{l}\text { Califique en escala de } 1 \text { (mínimo nivel de acuerdo) a } 7 \text { (máximo nivel de acuerdo) cada una de las siguientes } \\
\text { aseveraciones. Marque con una X donde corresponda su respuesta. Los datos serán tratados de manera general } \\
\text { preservando el anonimato de las respuestas individuales. }\end{array}$
\end{tabular}

1. La institución tiene un eficiente sistema de exploración de la información interna, tal que favorece la labor del equipo de alta dirección.

\begin{tabular}{l|l|l|l|l|l|l|}
\hline 1 & 2 & 3 & 4 & 5 & 6 & 7 \\
\hline $\begin{array}{l}\text { 2. La institución tiene un eficiente sistema de exploración de la información externa, tal que favorece la labor del } \\
\text { equipo de alta dirección. }\end{array}$
\end{tabular}
equipo de alta dirección.
\begin{tabular}{l|l}
1 & 2 \\
\hline
\end{tabular}
$2 \quad 3$
4
5
$6 \quad 7$

3. La institución tiene un sistema de información que le permite, a su equipo de alta dirección, identificar hallazgos importantes para su quehacer tanto de fuentes internas como externas.

\begin{tabular}{|c|c|c|c|c|c|c|}
\hline 1 & 2 & 3 & 4 & 5 & 6 & 7 \\
\hline
\end{tabular}

4. Los directivos de la institución crean nuevos conocimientos considerando el sistema de exploración, detección de hallazgos e integración de información.

\begin{tabular}{|c|c|c|c|c|c|c|c|}
\hline 1 & 2 & 3 & 4 & 5 & 6 & 7 \\
\hline 5. Los directivos de la institución & interactúan entre sí favoreciendo la creación de conocimiento. \\
\hline 1 & 2 & 3 & 4 & 5 & 6 & 7 \\
\hline 6. El equipo de alta dirección de la institución intercambia colaborativamente, conocimientos entre sí. \\
\hline 1
\end{tabular}

7. El equipo de alta dirección de la institución transforma el conocimiento individual en conocimiento grupal.

\begin{tabular}{|c|c|c|c|c|c|c|}
\hline 1 & 2 & 3 & 4 & 5 & 6 & 7 \\
\hline
\end{tabular}

8. El equipo directivo de la institución se transfiere colaborativamente, conocimientos mutuamente.

\begin{tabular}{|c|c|c|c|c|c|c|c|}
\hline 1 & 2 & 3 & 4 & 5 & 6 & 7 \\
\hline 9. Los directivos de la institución comparten colaborativamente conocimiento mutuamente entre sí. \\
\hline 1 & 2 & 3 & 4 & 5 & 6 & 7 \\
\hline 10. Los directivos de la institución aplican los conocimientos generados y compartidos. \\
\hline 1
\end{tabular}

11. Los directivos toman decisiones basados en la aplicación de conocimientos previamente generados y compartido.

\begin{tabular}{|c|c|c|c|c|c|c|}
\hline 1 & 2 & 3 & 4 & 5 & 6 & 7 \\
\hline 12. El conocimiento individual y grupal se transforma en conocimiento organizacional. \\
\hline 1 & 2 & 3 & 4 & 5 & 6 & 7 \\
\hline 13. El equipo de dirección basa su actuación en el conocimiento creado y compartido. \\
\hline 1
\end{tabular}

\section{Dimensiones, variables y medidas}

La dimensión crear conocimiento es medida por 4 ítems (Alpha de Cronbach=0,874); la dimensión compartir conocimiento es medida por 5 ítems (Alpha de Cronbach $=0,879$ ); y la dimensión aplicar conocimiento es medida por 5 ítems (Apha de Cronbach $=0,873$ ). Son ítems adaptados especialmente para instituciones universitarias a partir del trabajo de Rodríguez-Ponce (2007) y se emplea una escala Likert de 1 a 7. 


\section{Análisis de datos}

Los datos recogidos mediante el cuestionario fueron ingresados en la base de datos SPSS 21.0 para análisis estadístico. De este modo, se analiza las medias y las desviaciones estándar de cada dimensión. Seguidamente, se evalúan las diferencias de las medias de cada ítem respecto de la mediana de la escala empleada (valor 4,00).

\section{HALLAZGOS Y DISCUSIÓN}

\section{Hallazgo 1: Las dimensiones de la gestión del conocimiento se correlacionan entre sí}

En la muestra analizada se evidencian correlaciones significativas entre las diferentes fases de la gestión del conocimiento, obtenidos a partir de las percepciones de los directivos, tal como se muestra en la Tabla 2. Lo anterior sugiere que en la percepción de los directivos mientras mejor sea el proceso de crear conocimiento mejor será el proceso de compartir conocimiento (y viceversa); y también será mejor el proceso de aplicar conocimiento (y viceversa). Finalmente, en la percepción de los directivos un mejor proceso de compartir conocimiento se asocia con un mejor proceso de aplicar conocimiento (y viceversa). En consecuencia, el proceso de gestión del conocimiento desde la mirada o percepción de los directivos puede entenderse como un todo integrado, en donde existen fases independientes, pero altamente correlacionadas.

Tabla 2: Correlaciones entre las dimensiones de la gestión del conocimiento. ${ }^{* *}$. La correlación es significativa al nivel 0,01 (bilateral).

\begin{tabular}{|c|c|c|c|c|}
\hline \multicolumn{5}{|c|}{ Correlaciones } \\
\hline & & $\begin{array}{c}\text { Crear } \\
\text { conocimiento }\end{array}$ & $\begin{array}{c}\text { Compartir } \\
\text { conocimiento }\end{array}$ & $\begin{array}{c}\text { Aplicar } \\
\text { conocimiento }\end{array}$ \\
\hline \multirow{3}{*}{ Crear conocimiento } & Correlación de Pearson & 1 &, $886^{* *}$ &, $842^{* *}$ \\
\hline & Sig. (bilateral) & &, 000 &, 000 \\
\hline & $N$ & 34 & 34 & 34 \\
\hline \multirow{3}{*}{ Compartir conocimiento } & Correlación de Pearson &, $886^{* *}$ & 1 &, $913^{* *}$ \\
\hline & Sig. (bilateral) &, 000 & &, 000 \\
\hline & $N$ & 34 & 34 & 34 \\
\hline \multirow{3}{*}{ Aplicar conocimiento } & Correlación de Pearson &, $842^{* *}$ &, $913^{* *}$ & 1 \\
\hline & Sig. (bilateral) &, 000 &, 000 & \\
\hline & $N$ & 34 & 34 & 34 \\
\hline
\end{tabular}

Hallazgo 2: El promedio de las percepciones de la creación de conocimiento no varía significativamente de la mediana de la escala empleada

En la muestra analizada con las percepciones de los directivos, al comparar los valores obtenidos con la mediana de la escala, se obtienen los resultados de la Tabla 3.

Tabla 3: Resultados para la dimensión "crear conocimiento"

\begin{tabular}{|l|c|c|c|c|}
\hline \multicolumn{4}{|c|}{ Estadísticos descriptivos } & \\
\hline & $\mathrm{N}$ & Media & Desv. típ. & $\begin{array}{c}\text { Test de Media } \\
\text { (Mediana 4,00) }\end{array}$ \\
\hline $\begin{array}{l}\text { Sistema de exploración de fuentes internas y } \\
\text { externas }\end{array}$ & 34 & 3,735 & 1,377 & $-1,122$ \\
\hline Sistema de procesamiento de la información & 34 & 3,853 & 1,351 & $-0,634$ \\
\hline Sistemas de hallazgos & 34 & 3,735 & 1,263 & $-1,223$ \\
\hline Sistema de generación de conocimiento & 34 & 4,265 & 1,238 & $+1,224$ \\
\hline CREAR CONOCIMIENTO & 34 & 3,897 & 1,121 & $-0,352$ \\
\hline N válido (según lista) & 34 & & & \\
\hline
\end{tabular}


Así, desde la percepción de los directivos la creación de conocimiento muestra su valor promedio que no es estadísticamente significativo de la mediana de la escala $(4,00)$. Más aún, ninguno de los ítems que configuran la creación de conocimiento varía significativamente de la mediana de la escala.

Hallazgo 3: El promedio de las percepciones respecto del compartir conocimiento no varía significativamente de la mediana de la escala

En la muestra analizada al comparar los valores obtenidos con la mediana de la escala, se obtienen los resultados de la Tabla 4. Así, el compartir conocimiento desde la percepción de los directivos tiene un valor promedio que no es estadísticamente significativo de la mediana de la escala $(4,00)$. Sin embargo, existen 2 ítems que están estadísticamente por sobre la mediana. El primero se refiere al proceso de transferencia de conocimiento en los equipos de dirección de las unidades académicas, que es estadísticamente superior a la mediana de la escala empleada. El segundo ítem se refiere a la voluntad de los equipos por compartir el conocimiento transformado. Con todo, el hecho que los procesos de interacción, intercambio, y transformación no sean estadísticamente diferentes de la mediana, tiene la suficiente relevancia para establecer que el promedio de las percepciones de los directivos respecto del proceso de compartir conocimiento como un todo, no sea estadísticamente diferente de la mediana en la muestra analizada.

Tabla 4: Resultados para la variable compartir conocimiento ( ${ }^{*}$ significativo al 10\%)

\begin{tabular}{|c|c|c|c|c|}
\hline \multicolumn{4}{|c|}{ Estadísticos descriptivos } & \multirow[b]{2}{*}{$\begin{array}{l}\text { Test de Media } \\
\text { (Mediana 4,00) }\end{array}$} \\
\hline & $\mathrm{N}$ & Media & Desv. típ. & \\
\hline $\begin{array}{l}\text { Proceso de interacción entre los } \\
\text { miembros en el manejo del } \\
\text { conocimiento }\end{array}$ & 34 & 4,265 & 1,287 & $+1,200$ \\
\hline $\begin{array}{l}\text { Proceso de intercambio de } \\
\text { conocimiento }\end{array}$ & 34 & 3,882 & 1,274 & $-0,538$ \\
\hline $\begin{array}{l}\text { Proceso de transformación del } \\
\text { conocimiento }\end{array}$ & 34 & 3,706 & 1,382 & $-1,240$ \\
\hline $\begin{array}{l}\text { Proceso de transferencia de } \\
\text { conocimiento }\end{array}$ & 34 & 4,3824 & 1,18103 & $+1,889$ * \\
\hline $\begin{array}{l}\text { Voluntad para compartir el } \\
\text { conocimiento transformado }\end{array}$ & 34 & 4,7353 & 1,13642 & $+3,773^{\star * *}$ \\
\hline Compartir Conocimiento & 34 & 4,1941 & 1,07024 & $+1,057$ \\
\hline N válido (según lista) & 34 & & & \\
\hline
\end{tabular}

Hallazgo 4: El promedio de las percepciones respecto del aplicar conocimiento no varía significativamente de la mediana de la escala empleada

En la muestra analizada al comparar los valores obtenidos con la mediana de la escala, se obtienen los resultados de la Tabla 5. Así, el aplicar conocimiento como una dimensión exhibe un valor promedio de las percepciones de los directivos que es inferior de manera estadísticamente significativa de la mediana de la escala $(4,00)$.

Ciertamente, existen 3 ítems cuyo promedio de las percepciones de los directivos no es estadísticamente significativo de la mediana. Son los ítems referidos a la aplicación de conocimientos, el empleo de los mismos en los procesos de toma de decisiones, y la incorporación de los conocimientos a la organización. Sin embargo, 2 ítems son inferiores estadísticamente a la mediana de la escala, éstos se refieren a si los directivos basan sus actuaciones en el conocimiento, y si el conocimiento aplicado crea o no valor estratégico para la unidad académica. La magnitud de estos últimos ítems y su impacto en el promedio de la dimensión denominada aplicar conocimiento, hacen que esta fase final del proceso sea significativamente inferior a la mediana de la escala empleada.

En resumen, es posible establecer que de acuerdo con las percepciones de los directivos encuestados, las dimensiones que configuran la gestión del conocimiento se correlacionan entre sí de manera significativa. De esta forma, cada una de las fases de la gestión del conocimiento se relaciona de manera directa con las 
otras fases. Este descubrimiento sugiere que no es posible considerar cada fase de manera aislada, sino que la gestión del conocimiento es un proceso global que requiere una consideración integradora. Estos resultados son consistentes con otras investigaciones realizadas en Chile pero en pequeñas y medianas empresas (Pedraja-Rejas y Rodríguez-Ponce, 2008) y en organizaciones públicas (Rodríguez-Ponce, 2007), las que muestran altos grados de correlación entre las fases de crear, compartir y aplicar conocimiento.

Tabla 5: Resultados para la dimensión "aplicar conocimiento"

\begin{tabular}{|l|c|c|c|c|}
\hline \multicolumn{4}{|c|}{ Estadísticos descriptivos } & N \\
\hline $\begin{array}{l}\text { Directivos aplican los conocimientos } \\
\text { compartidos }\end{array}$ & 34 & 3,853 & $\begin{array}{c}\text { Desv. } \\
\text { típ. }\end{array}$ & $\begin{array}{c}\text { Test de Media } \\
\text { (Mediana 4,00) }\end{array}$ \\
\hline $\begin{array}{l}\text { La toma de decisiones se basa en la } \\
\text { aplicación de conocimiento }\end{array}$ & 34 & 3,851 & 1,579 & $-0,615$ \\
\hline $\begin{array}{l}\text { El conocimiento se aplica y pasa a ser } \\
\text { incorporado a la organización }\end{array}$ & 34 & 3,7941 & 1,27397 & $-0,550$ \\
\hline $\begin{array}{l}\text { Las actuaciones de los directivos se basan } \\
\text { en el conocimiento aplicado }\end{array}$ & 34 & 3,1765 & 1,38105 & $-3,477^{\star * *}$ \\
\hline $\begin{array}{l}\text { El conocimiento aplicado genera servicios, } \\
\text { productos o crear valor para la unidad. }\end{array}$ & 34 & 2,9412 & 1,34708 & $-4,583^{\star * *}$ \\
\hline Aplicar Conocimiento & 34 & 3,524 & 1,13886 & $-2,439^{\star *}$ \\
\hline N válido (según lista) & 34 & & & \\
\hline
\end{tabular}

Sin embargo, este es el primer estudio, si bien de carácter exploratorio, en el campo de las universidades chilenas que propone este hallazgo. Hallazgo que debe ser analizado en un estudio con una metodología y un tamaño muestra que permita inferencia estadística. Ciertamente, no es trivial considerar que el proceso de gestión del conocimiento puede entenderse como un todo más que como fases separadas, ya que la sugerencia práctica que emerge es que la cadena de la gestión del conocimiento es tan débil como su eslabón más frágil. Los alcances de este resultado pueden ser muy importantes ya que ponen de manifiesto que la gestión del conocimiento requiere esfuerzos permanentes, sistemáticos e ininterrumpidos por parte de la alta dirección.

Una mirada a las dimensiones sugiere, en la muestra analizada, que el promedio de las percepciones de los directivos del crear y compartir conocimiento (como una dimensión global) no muestra diferencias significativas con la mediana de la escala empleada. Sin embargo, en un análisis pormenorizado se descubre que la transferencia de conocimiento en los equipos de dirección de las unidades académicas y la voluntad de los equipos por compartir el conocimiento transformado son valores que están significativamente por sobre el promedio de la escala empleada. Este es un descubrimiento novedoso ya que no existen investigaciones equivalentes en instituciones universitarias chilenas.

Finalmente, se halla que la aplicación de conocimiento, como un todo, exhibe un promedio por debajo de la mediana de la escala empleada. En efecto, según la percepción de quienes responden el cuestionario, las actuaciones de los directivos no se basan en el proceso de gestión del conocimiento llevado a cabo. Además, en sus percepciones los directivos visualizan que el conocimiento aplicado no crea un gran valor estratégico para la unidad académica. Probablemente, sea muy difícil crear valor estratégico con actuaciones no alineadas al proceso previo de gestión del conocimiento.

Este es un hallazgo novedoso por la inexistencia de estudios equivalentes en Chile. Pero en la literatura internacional se ha probado reiteradamente que la aplicación del conocimiento es una fase determinante del éxito en la gestión del conocimiento (Sarin y McDermott, 2003) y su culminación es difícil ya que un proceso previo de crear y compartir conocimiento es condición necesaria pero no suficiente para aplicar conocimiento con éxito (Choi et al., 2010).

\section{CONCLUSIONES}

La economía de las organizaciones ha evolucionado con fuerza a partir del trabajo seminal (1937) del Premio Nobel de Economía Ronald Coase, cuyas bases conceptuales se han nutrido con múltiples aportaciones destacando desde luego las notables contribuciones de los también Premios Nobel de 
Economía Herbert Simon y Oliver Williamson. En lo fundamental, las organizaciones son esenciales y se conforman por agentes con relaciones permanentes más allá de las transacciones de mercado, pero estos agentes poseen una racionalidad limitada, es decir, no son capaces de visualizar, ni de evaluar todas las alternativas que existen en la resolución de un problema. Más aún, su base cognitiva y sus valores pueden afectar y sesgar las decisiones a diseñar e implementar.

Por lo mismo, la gestión del conocimiento en los equipo de alta dirección puede ser una variable que marque la diferencia en la calidad de las decisiones y en los resultados de las organizaciones. Esta lógica o perspectiva de análisis es plausible de aplicar en el caso de las universidades que también son organizaciones, tienen agentes con relaciones permanentes y equipos de dirección.

En esta investigación de carácter exploratorio es posible inducir, a partir de las percepciones de los directivos, algunos hallazgos y conclusiones muy relevantes con claras implicancias prácticas para la gestión universitaria:

La gestión del conocimiento tiene fases que teóricamente se pueden separar, pero que en términos prácticos son indisolubles entre sí por su alto grado de correlación. Por consiguiente, la cadena que se logra gestionando el conocimiento será tan fuerte como su eslabón más débil.

En las universidades analizadas la fase de aplicar conocimiento exhibe un promedio que es significativamente más bajo que la mediana de la escala empleada, lo que sugiere una mirada especial en esta fase puesto que particularmente lo que dice relación con: que las actuaciones de los directivos no se basan necesariamente en la aplicación de conocimiento; y el conocimiento aplicado no necesariamente genera servicios, productos o crear valor para la unidad académica.

Ambos aspectos son de gran importancia puesto que dejan de manifiesto, que en la percepción de los directivos, existen aspectos que se deben mejorar para lograr una gestión del conocimiento de mayor calidad.

Ciertamente, se debe indicar que como toda investigación de carácter exploratorio no se pretende en este trabajo realizar inferencia estadística ni deducir principios generales. Lo cual no obsta para contribuir al estado del arte con: evidencia empírica, validación de una escala de medida, y generación de puntos de atención o alerta para mejorar la gestión del conocimiento. Con todo, este estudio empírico da soporte a la teoría de los escalafones superiores ya que existen variaciones significativas en las percepciones de los directivos respecto de los procesos, lo cual sugiere que un mismo proceso de gestión del conocimiento es visto de forma diferente por distintos actores y/o que los procesos de gestión del conocimiento entre universidades son distintos.

\section{AGRADECIMIENTOS}

Los autores agradecen el patrocinio de la Comisión Nacional de Investigación Científica y Tecnológica de Chile, CONICYT a través del proyecto FONDECYT № 1140026: "Calidad de las instituciones de Educación Superior: El impacto de la gestión del conocimiento y los estilos de liderazgo". Asimismo se agradecen los valiosos comentarios de 3 árbitros anónimos, sugerencias que permitieron un mejoramiento significativo de la primera versión de este trabajo. Sin embargo, los errores que puedan existir son de exclusiva responsabilidad de los autores.

\section{REFERENCIAS}

Alegre, J.; K. Sengupta y R. Lapiedra, Knowledge management and innovation performance in a high-tech SMEs industry, International Small Business Journal: 31(4), 454-470 (2013)

Amit, R. y P. Schoemaker, Strategic assets and organizational rent, Strategic management journal: 14(1), 33-46 (1993)

Araneda-Guirriman, C. A., E. Rodríguez-Ponce y L. Pedraja-Rejas, Relación entre el Financiamiento Fiscal, la Calidad del Cuerpo Académico y la Retención de Estudiantes Universitarios en Chile. Formación Universitaria, 6(6), 55-64 (2013)

Chaudhri, V.K.; N. Dinesh y D. Inclezan, Three lessons for creating a knowledge base to enable explanation, reasoning and dialog, en Proceedings of the Second Annual Conference on Advances in Cognitive Systems ACS: 187-203 (2013) 
Choi, S.Y.; H. Lee y Y. Yoo, The Impact of Information Technology and Transactive Memory Systems on Knowledge Sharing, Application, and Team Performance: A Field Study, MIS quarterly: 34(4), 855-870 (2010)

Chong, C.W. y J. Besharati, Challenges of knowledge sharing in the petrochemical industry, Knowledge Management \& E-Learning (KM\&EL): 6(2), 171-187 (2014)

Dalkir K. Knowledge Management in Theory and Practice. Routledge. (2013)

Gairín, J., Promover y gestionar el conocimiento colectivo para mejorar la cultura y la práctica de la seguridad en educación, Cuadernos de estrategia: (172), 59-84 (2015)

Geisler, E. y N. Wickramasinghe, Principles of knowledge management: Theory, practice, and cases. Routledge, NY, USA (2015)

Goldman, S.R. y M. Scardamalia, Managing, understanding, applying, and creating knowledge in the information age: Next-generation challenges and opportunities, Cognition and Instruction: 31(2), 255-269 (2013)

Grant, R.M., Toward a knowledge-based theory of the firm. Strategic management journal: 17(2), 109-122 (1996)

Hume, C., P. Clarke y M. Hume, The role of knowledge management in the large non profit firm: building a framework for KM success, International Journal of Organisational Behaviour: 17(3), $82-104$ (2012)

Kumar, S.A., Knowledge management and new generation of libraries information services: a concepts. International Journal of Library and Information Science: 1(2), 24-30 (2010)

Lee, K.C., S. Lee y I. Kang, KMPI: measuring knowledge management performance, Information \& management: 42(3), 469-482 (2005)

Lee, M.R. y T.T. Chen, Revealing research themes and trends in knowledge management: From 1995 to 2010, Knowledge-Based Systems: 28, 47-58 (2012)

López, S.G., J.L. Benítez y J.M. Sánchez, Social Knowledge Management from the Social Responsibility of the University for the Promotion of Sustainable Development, Procedia-Social and Behavioral Sciences: 191, 2112-2116 (2015)

Martín de Castro, G., Knowledge management and innovation in knowledge-based and high-tech industrial markets: The role of openness and absorptive capacity, Industrial Marketing Management: 47, 143-146 (2015)

Martins, J.B. y R.C. Miranda, Improvement of strategists and novice decision makers: strategic knowledge management applied to the Technology Development Support Center at the Universidade de Brasilia, Brazil, Transinformação: 23(2), 139-157 (2011)

Nonaka, I. y H. Takeuchi, The knowledge-creating company: How Japanese companies create the dynamics of innovation. Oxford university press, NY, USA (1995)

Osei-Bryson, K.M.; G. Mansingh y L. Rao, Understanding and Applying Knowledge Management and Knowledge Management Systems in Developing Countries: Some Conceptual Foundations, in Knowledge Management for Development by Osei-Bryson, Mansingh y Rao, pp. 1-15. Springer, USA (2014)

Osmani, M.; A.R. Zaidi y M. Nilashi, Motivational Factors, Trust and Knowledge Sharing in Organizations, International Journal of Innovation and Scientific Research: 12(2), 463-474 (2014)

Patel, V.K. y A.B. Gorvadiya, Knowledge management: the need of modern organizations, Journal of Knowledge Management: 6(3), 259-261 (2014)

Pedraja-Rejas, L. y E. Rodríguez-Ponce, Estilos de liderazgo, gestión del conocimiento y diseño de la estrategia: un estudio empírico en pequeñas y medianas empresas, Interciencia: Revista de ciencia y tecnología de América: 33(9), 651-657 (2008) 
Pedraja-Rejas, L.; E. Rodríguez-Ponce y J. Rodríguez-Ponce, Sociedad del conocimiento y dirección estratégica: Una propuesta integradora, Interciencia: Revista de ciencia y tecnología de América, 31(8), 570$576(2006)$

Prahalad, C.K. y G. Hamel, The core competence of the corporation, Harvard Business Review: 68(3), 7991 (1990)

Ritala, P.; H. Olander; S. Michailova y K. Husted, Knowledge sharing, knowledge leaking and relative innovation performance: An empirical study, Technovation: 35, 22-31 (2015)

Rodríguez-Gómez, D. y J. Gairín, Innovación, aprendizaje organizativo y gestión del conocimiento en las instituciones educativas, Educación: 24(46), 73-90 (2015)

Rodríguez, E. La gestión del conocimiento en los equipos directivos de las universidades y sus efectos sobre el sistema de aseguramiento de la calidad institucional: evidencia empírica desde Chile. Tesis doctoral. Universidad Autónoma de Barcelona. (2012)

Rodríguez-Ponce, E., El rol de las universidades en la sociedad del conocimiento y en la era de la globalización: evidencia desde Chile, Interciencia: Revista de ciencia y tecnología de América, 34(11), 822829 (2009)

Rodríguez-Ponce, E., Gestión del conocimiento y eficacia de las organizaciones un estudio empírico en instituciones públicas, Interciencia: Revista de ciencia y tecnología de América, 32(12), 820-826 (2007)

Rodríguez-Ponce, E.; W. Cohen-Hornickel; L. Pedraja-Rejas; C. Araneda-Guirriman y J. Rodríguez-Ponce, La gestión del conocimiento y la calidad de la docencia de postgrado en las universidades: un estudio exploratorio, Innovar: Revista de Ciencias Administrativas y Sociales, 24(52), 59-66 (2014)

Sarin, S. y C. McDermott, The effect of team leader characteristics on learning, knowledge application, and performance of cross-functional new product development teams, Decision sciences: 34(4), 707-739 (2003)

Simon, H. A. Rational Decision Making in Business Organizations. American Economic Review. 69(4), 493513 (1979)

Smith, A.D. y W.T. Rupp, Communication and loyalty among knowledge workers: a resource of the firm theory view, Journal of knowledge management: 6(3), 250-261 (2002)

Snow, C. C. y J.B. Thomas, Field research methods in strategic management: contributions to theory building and testing. Journal of Management Studies, 31, 457-480 (1994)

Stone, D.L., Creating knowledge that makes important contributions to society, Journal of Managerial Psychology: 25(3), 192-200 (2010)

Wang, Z., N. Wang y $\mathrm{H}$. Liang, Knowledge sharing, intellectual capital and firm performance, Management decision: 52(2), 230-258 (2014)

Wernerfelt, B., A resource-based view of the firm, Strategic management journal: 5(2), 171-180 (1984)

Williamson, O.E., The Theory of the Firm as Governance Structure: From Choice to Contract. Journal of Economic Perspectives 16(3), 171-195 (2002)

Zhao, Y. y M. Lavin, An empirical study of knowledge transfer in working relationships with suppliers in new product development, International Journal of Innovation Management: 16(02), 1-26 (2012) 
